Case

Report

\title{
A Case of Castleman's Disease with Myasthenia Gravis
}

\author{
Keidai Ishikawa, MD, PhD, ${ }^{1,2}$ Tatsuya Kato, MD, PhD, ${ }^{1,2}$ Masato Aragaki, MD, ${ }^{1}$ \\ Toshiro Ohbuchi, MD, ${ }^{3}$ Sachiko Kimura, MD,${ }^{4}$ Yoshiro Matsui, MD, PhD, ${ }^{2}$ \\ and Mitsuhito Kaji, $\mathrm{MD}, \mathrm{PhD}^{1}$
}

\begin{abstract}
A rare case of Castleman's disease with myasthenia gravis is reported. A 55-year-old woman with bilateral ptosis, speech impairment, and severe dyspnea had been previously diagnosed with myasthenia gravis. Computed tomography showed a $5 \mathrm{~cm} \times 3 \mathrm{~cm}$ paratracheal mass in the mediastinum, thought to be an ectopic thymoma. Two days after surgical resection, the patient suddenly developed dyspnea. Postoperative myasthenic crisis was diagnosed, and plasmapheresis was performed. Her general condition improved, and her subsequent course was uneventful. The final pathological diagnosis was mediastinal solitary Castleman's disease, hyaline vascular type. Castleman's disease with myasthenia gravis is especially rare. One of the serious complications is postoperative myasthenic crisis. For patients with myasthenia gravis, the rate of postoperative myasthenic crisis seems significantly higher in Castleman's disease patients than in patients with thymic epithelial tumors. Castleman's disease with myasthenia gravis is discussed along with a review of the literature.
\end{abstract}

Keywords: castleman's disease, giant lymph node hyperplasia, mediastinal tumor, myasthenia gravis, postoperative myasthenic crisis

\section{Introduction}

Castleman's disease is an atypical giant lymph node hyperplasia of uncertain origin. Most lesions are located in the chest. The condition is commonly associated with

\footnotetext{
${ }^{1}$ Department of Thoracic Surgery, Sapporo Minami-Sanjo Hospital, Sapporo, Hokkaido, Japan

${ }^{2}$ Department of Cardiovascular and Thoracic Surgery, Hokkaido University Graduate School of Medicine, Sapporo, Hokkaido, Japan ${ }^{3}$ Department of Thoracic Surgery, Sapporo Minami-Ichijo Hospital, Sapporo, Hokkaido, Japan

${ }^{4}$ Department of Pathology, Sapporo Medical University Hospital, Sapporo, Hokkaido, Japan
}

Received: October 1, 2012; Accepted: January 15, 2013 Corresponding author: Keidai Ishikawa, MD, PhD. Department of Thoracic Surgery, Sapporo Minami-Sanjo Hospital, S3W6 Chuo-ku, Sapporo, Hokkaido 060-0063, Japan Email: keidaiishikawa@hotmail.com (C)2014 The Editorial Committee of Annals of Thoracic and Cardiovascular Surgery. All rights reserved. clinical abnormalities, including POEMS syndrome (polyneuropathy, organomegaly, endocrinopathy, monoclonal gammopathy, and skin changes), paraneoplastic pemphigus, and Hodgkin's disease, but rarely with myasthenia gravis.

A rare case of a Castleman's disease patient with myasthenia gravis is presented along with a review of the literature.

\section{Case Report}

A previously healthy, 55-year-old woman was transferred to our hospital for resection of an upper mediastinal tumor in 2003. She initially presented with bilateral ptosis, speech impairment, and severe dyspnea, and she had been previously diagnosed with acetylcholine receptorpositive myasthenia gravis. She was having corticosteroid treatment. A computed tomography (CT) scan revealed a $5 \mathrm{~cm} \times 3 \mathrm{~cm}$, well-circumscribed, paratracheal mass 


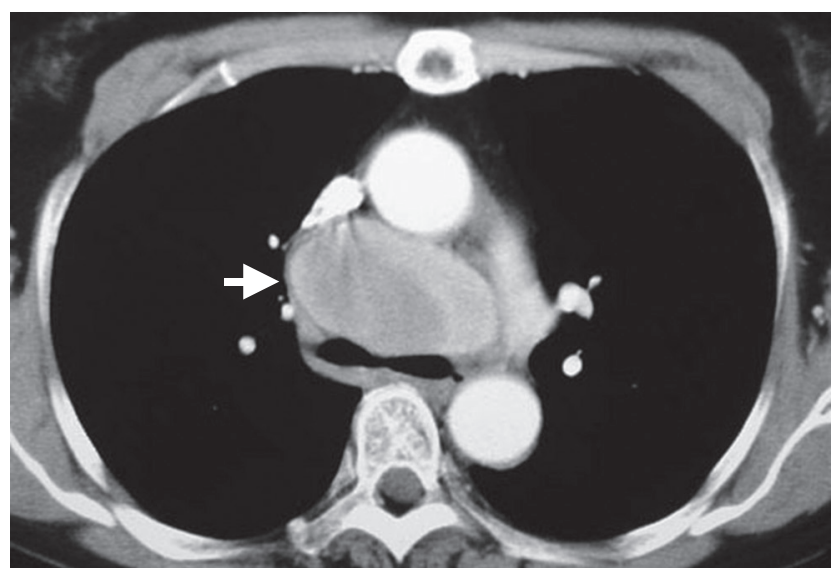

Fig. 1 Computed tomography (CT) scan of the chest showing a well-circumscribed, right paratracheal mass (arrow).

(Fig 1) in the mediastinum, thought to be an ectopic thymoma. On her Admission, She had mild bilateral ptosis without respiratory distress.

At operation, video-assisted thoracic surgery (VATS) was performed. With the patient positioned in the left lateral decubitus position, a 35-mm, right mini-thoracotomy was performed with VATS in the fourth intercostal space, and an additional 10-mm port was inserted in the sixth intercostal space at the anterior axillary line, without using a rib retractor. The thymus was found to be intact. The middle mediastinal tumor was located between the superior vena cava and the trachea. The mass was adherent to adjacent structures, particularly the superior vena cava and trachea. The right vagus nerve was located across the mass. The right vagus nerve was identified and preserved, and the mass was carefully dissected from the upper mediastinum. After successful removal of the mass and adequate hemostasis, the chest was closed. Two days after the operation, the patient experienced rapid onset of dyspnea. Her Tensilon test was positive, and she was diagnosed as having a postoperative myasthenic crisis, and mechanical ventilation with orotracheal intubation, plasmapheresis, additional corticosteroid treatment, and 3 days of immunoglobulin therapy were given. Her general condition improved. Seven days after treatment, she could breathe without the assistance of mechanical ventilation, and the patient's postoperative course after treatment for the myasthenic crisis was uneventful.

A surgical specimen of the tumor was sent for histologic analysis. The tumor was enclosed in a fibrous capsule, and the cut surface was white lymphadenoid tissue, with leiomyoma-like nodules. These nodules originated from lymph nodes, not from the thymus. Proliferating

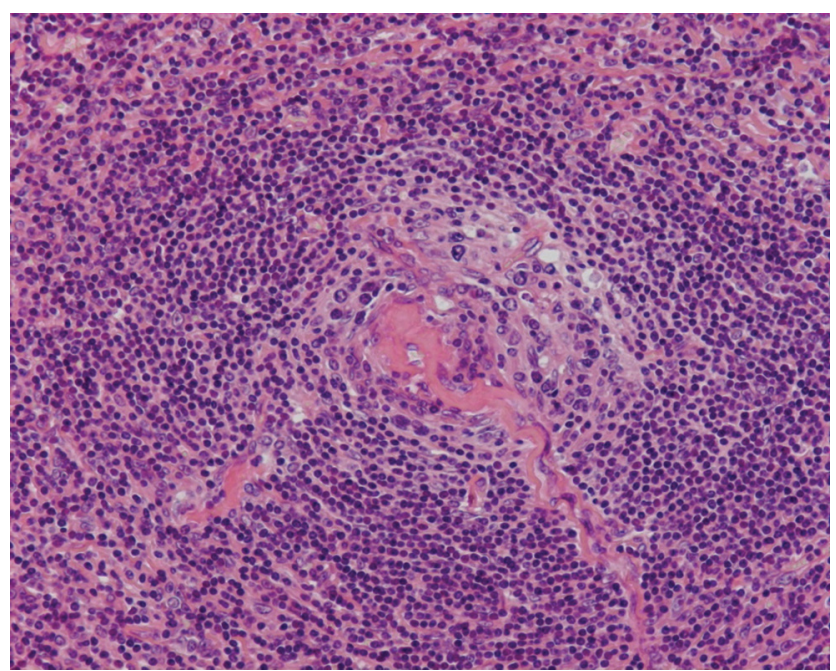

Fig. 2 Photomicrograph of a tumor section. At high magnification (hematoxylin and eosin, $\times 200$ ), the hyaline vascular pattern is recognizable. The follicles, containing a central irregular area with a peripheral cuff of lymphocytes, are readily apparent.

spindle cells were seen around these nodules. The removed mass was composed of lymphoid tissue with follicles containing spindle cells.

Postcapillary venules were prominent at the periphery of the follicles, which were surrounded by centric cuffs of lymphocytes arranged in an onion-skin pattern (Fig. 2). Immunohistochemically, the tumors were positive for vimentin and alpha-smooth muscle actin. These features were consistent with a diagnosis of mediastinal solitary Castleman's disease of hyaline vascular type. No evidence of recurrence has been found over 96 months of follow-up.

\section{Discussion}

Castleman's disease is often referred to as giant lymph node hyperplasia or angiofollicular lymph node hyperplasia. It was first described by Castleman in 1956 as an atypical lymphoproliferative disorder of uncertain origin. ${ }^{1)}$ Most lesions are located in the chest, along the tracheobronchial tree, in the mediastinum, or at the lung hilus. Patients are usually asymptomatic or have nonspecific complaints, such as cough, dyspnea, chest pain, or back pain, which are often due to tracheobronchial compression.

The disease can be classified on the basis of histologic and clinical characteristics (solitary or multicentric disease). Three histologic types have been described: hyaline vascular, plasma cell, and a mixed type. ${ }^{2)}$ It is becoming 
Table 1 Summary of reports in the literature of Castleman's disease with myasthenia gravis

\begin{tabular}{lcccllclc}
\hline Reference & Year & Age & Sex & Tumor location & Clinical type & Tumor size (cm) & Histological type & Crisis* \\
\hline Emson, et al. ${ }^{8}$ & 1973 & $14 \mathrm{y}$ & $\mathrm{F}$ & retroperitoneum & solitary & $4.5 \times 3.5 \times 2.3$ & hyaline vascular & Yes \\
Pasaoglu, et al. $^{9}$ & 1994 & $15 \mathrm{y}$ & $\mathrm{F}$ & neck, mediastinum & multicentric & $8 \times 8 \times 6$ & mixed-cell & No \\
Chorzeiski, et al. $^{10}$ & 1999 & $31 \mathrm{y}$ & $\mathrm{M}$ & retroperitoneum & solitary & $14 \times 10.6 \times 8$ & hyaline vascular & No \\
Day, et al. $^{11}$ & 2003 & $39 \mathrm{y}$ & $\mathrm{F}$ & mediastinum & solitary & $5.5 \times 4.5 \times 2.5$ & hyaline vascular & No \\
Westphal, et al. $^{12}$ & 2010 & $72 \mathrm{y}$ & $\mathrm{F}$ & mediastinum & solitary & not described & hyaline vascular & No \\
Kojima, et al. $^{13}$ & 2011 & $59 \mathrm{y}$ & $\mathrm{M}$ & mediastinum & solitary & 10 & hyaline vascular & No \\
Lee, et al. $^{14}$ & 2012 & $51 \mathrm{y}$ & $\mathrm{M}$ & mediastinum & solitary & $7 \times 6$ & hyaline vascular & Yes \\
Present study & 2013 & $55 \mathrm{y}$ & $\mathrm{F}$ & mediastinum & solitary & $5 \times 3 \times 2.5$ & hyaline vascular & Yes \\
\hline
\end{tabular}

*: Postoperative myasthenic crisis

increasingly clear that the different subtypes have different etiologies. There have been reports of patients with Castleman's disease with various associated clinical abnormalities, included POEMS syndrome, ${ }^{3)}$ paraneoplastic pemphigus, ${ }^{4)}$ and Hodgkin's disease. ${ }^{5)}$

Treatment for Castleman's disease varies depending on the histologic and clinical features of the particular case. Simple excision of solitary nodules is usually curative, especially for the hyaline vascular type of disease. ${ }^{6)}$ The plasma cell type is frequently associated with systemic manifestations, such as amyloidosis, and is often refractory to systemic therapy with corticosteroids and chemotherapy, particularly when it is multicentric.6) Recently, in clinical trials, it has been found that anti-IL-6 receptor antibody is effective for the treatment of Castleman's disease. ${ }^{6}$ )

Myasthenia gravis and thymic epithelial tumors are both associated with acetylcholine receptor antibody. Recently, however, muscle-specific kinase (MuSK) has also been associated with myasthenia gravis.7) Rabbits immunized with MuSK ectodomain protein developed myasthenia gravis-like muscle weakness with a reduction in acetylcholine receptors clustering at the neuromuscular junctions. It was suggested that the MuSK may function via an alternative pathway linked to myasthenic syndrome that is different from the pathway of the acetylcholine receptor antibody.7)

Seven previous cases of Castleman's disease associated with myasthenia gravis, along with myasthenic crisis, which is one of the most serious postoperative complications of Castleman's disease, have been reported in the literature (Table 1), ${ }^{8-14)}$ In one study, 11 (5.4\%) of a total of 204 patients with surgically treated thymic epithelial tumors experienced postoperative myasthenic crisis. ${ }^{15)}$ Of the eight patients described in the literature with Castleman's disease and myasthenia gravis (including the present patient), three patients (37.5\%) experienced postoperative myasthenic crisis. For patients with myasthenia gravis, the rate of postoperative myasthenic crisis seems significantly higher in patients with Castleman's disease than in patients with thymic epithelial tumors. In both the reported patients who experienced postoperative myasthenic crisis, postoperative plasmapheresis was administered, which brought about dramatic improvements. Batocchi et al. recommended that myasthenic patients with oropharyngeal or respiratory muscle weakness receive preoperative plasma exchange to achieve a minimum level of muscle strength to prevent severe postoperative complications. ${ }^{16)}$

Although patients with Castleman's disease are usually asymptomatic or have nonspecific complaints if they also have myasthenia gravis and undergo surgical treatment, their chance of developing postoperative myasthenic crisis seems to be higher than that of patients with myasthenia gravis and thymic epithelial tumor. Since Castleman's disease with myasthenia gravis is rare, these assumptions are based on a limited number of previous cases.

\section{Conclusion}

Castleman's disease with myasthenia gravis is a rare condition. Castleman's disease itself is a benign disease histologically, but postoperative myasthenic crisis is a serious complication. For patients with myasthenia gravis, the rate of postoperative myasthenic crisis seems significantly higher in patients with Castleman's disease than in patients with thymic epithelial tumors. Plasmapheresis is likely to be effective for the treatment of postoperative myasthenic crisis in these cases.

\section{Disclosure Statement}

Keidai Ishikawa and co-authors have no conflicts of interest to declare. 


\section{References}

1) Castleman B, Iverson L, Menendez VP. Localized mediastinal lymphnode hyperplasia resembling thymoma. Cancer 1956; 9: 822-30.

2) Frizzera G. Castleman's disease and related disorders. Semin Diagn Pathol 1988; 5: 346-64.

3) Adelman HM, Cacciatore ML, Pascual JF, et al. Case report: Castleman disease in association with POEMS. Am J Med Sci 1994; 307: 112-4.

4) Caneppele S, Picart N, Bayle-Lebey P, et al. Paraneoplastic pemphigus associated with Castleman's tumour. Clin Exp Dermatol 2000; 25: 219-21.

5) Abdel-Reheim FA, Koss W, Rappaport ES, et al. Coexistence of Hodgkin's disease and giant lymph node hyperplasia of the plasma-cell type (Castleman's disease). Arch Pathol Lab Med 1996; 120: 91-6.

6) Nishimoto N, Sasai M, Shima Y, et al. Improvement in Castleman's disease by humanized antiinterleukin-6 receptor antibody therapy. Blood 2000; 95: 56-61.

7) Shigemoto K, Kubo S, Maruyama N, et al. Induction of myasthenia by immunization against muscle-specific kinase. J Clin Invest 2006; 116: 1016-24.

8) Emson HE. Extrathoracic angiofollicular lymphoid hyperplasia with coincidental myasthenia gravis. Cancer 1973; 31: 241-5.
9) Pasaoglu I, Dogan R, Topcu M, et al. Multicentric angiofollicular lymph-node hyperplasia associated with myasthenia gravis. Thorac Cardiovasc Surg 1994; 42: 253-6.

10) Chorzelski T, Hashimoto T, Maciejewska B, et al. Paraneoplastic pemphigus associated with Castleman tumor, myasthenia gravis and bronchiolitis obliterans. J Am Acad Dermatol 1999; 41: 393-400.

11) Day JR, Bew D, Ali M, et al. Castleman's disease associated with myasthenia gravis. Ann Thorac Surg 2003; 75: 1648-50.

12) Westphal FL, Lima LC, Santana LC, et al. Castleman's disease associated with follicular dendritic cell sarcoma and myasthenia gravis. J Braz Pneumol 2010; 36: 819-23. [Article in English, Portuguese]

13) Kojima M, Shimizu K, Kaneko Y, et al. Lymphoid hyperplasia of the thymus showing Castleman's disease in a patient with myasthenia gravis. Rheumatol Int 2012; 32: 3695-7. Epub 2011 Sep 27.

14) Lee SK, Kim DH, Son BS. Castleman's Disease with Myasthenia Gravis. Korean J Thorac Cardiovasc Surg 2012; 45: 199-201.

15) Fang W, Chen W, Chen G, et al. Surgical management of thymic epithelial tumors: a retrospective review of 204 cases. Ann Thorac Surg 2005; 80: 2002-7.

16) Batocchi AP, Evoli A, Di Schino C, et al. Therapeutic apheresis in myasthenia gravis. Ther Apher 2000; 4: 275-9. 